\title{
Assessment of an oral health scoring system by GDPs
}

\author{
Evaluation of an oral health scoring system by dentists in general dental practice F. J. T Burke, M. Busby,
}

\author{
S. McHugh, S. Delargy, A. Mullins and R. Matthews Br Dent J 2003; 194: 215-218
}

An Oral Health Index (OHX) has been designed to provide a numerical measure of the overall state of a patient's oral health by means of a series of simple clinical examinations. This has been amended to produce the Oral Health Score (OHS).

\section{Objective}

To assess, by means of a questionnaire, the ease of use and understanding of the OHS by general dental practitioners.

\section{Methods}

350 GDPs were asked to participate in the project, of whom 329 agreed. These dentists were given a lecture of 2 hours' duration on the OHS. The participating dentists were requested to use the OHS for a period of 1 year. At the end of this period, a questionnaire was delivered to them by post, with an explanatory letter and reply-paid envelope.

\section{Results}

Completed, useable questionnaires were received from 239 GDPs, a $77 \%$ response rate. Ninety three per cent of respondents considered the OHS instructions to be satisfactory. The respondents' views on the criteria on which the OHS component assessments are based indicated that over 90\% of respondents agreed with the criteria for caries, adequacy of restorations, periodontal assessment, mucosal assessment and assessment of dentures. A majority of respondents agreed that the OHS provided a valid representation of oral health.

\section{Conclusion}

The results of the present study indicate that the OHS is considered to be an easy-to-use measure of a patient's oral health and that it provides a valid representation of a patient's oral health.

\section{IN BRIEF}

- An oral health scoring system (the Denplan Oral Health Score) has been used by UK general dental practitioners in the examination of 140,000 patients.

- Respondents indicated general satisfaction with the concept of the Oral Health Score (OHS).

- Respondents indicated general agreement with most of the criteria used for the various parts of the examination.

- The OHS has also been used as a potential patient motivational tool.

\section{COMMENT}

The potential of a standardised method of describing a person's overall dental health status is immense. The authors of this report identify these as:

- A means of patient motivation.

- Framing a level of oral health that could be considered optimal.

- Measuring changes in oral health (including outcome measures).

- Assessing consistency in the evaluation of oral health by dentists.

- A means of measuring the oral health of populations and for service planning, evaluation and cost effectiveness of dental care

- A marketing tool for dental practices.

- A quality control tool.

- A basis for the prosecution and/or defence in litigation involving dental care.

I would perhaps take issue with the suggestion that the OHS system described in the paper might be a competitor to the Oral Health Impact Profile and similar measures of how oral health is perceived by people as affecting their lives. The view of some respondents in the survey that 'patient perception' be given a reduced weighting in the index is an indication that the dentists who evaluated it do not perceive OHS as a measure of subjective impact. The OHS is therefore perhaps best viewed as a composite measure of clinical status and as such fills a niche which to date, has yet to produce a widely accepted and used measure. This is an important and valuable contribution to a dimension of the assessment of dental clinical care with enormous potential. Although, as the authors make clear, there are still some aspects of the weighting system such as that concerned with periodontal health that perhaps need to be addressed. Nevertheless this could be a very valuable tool and we should perhaps look forward to the point where its development might enable it, for example, to be used to objectively assess the relative outcomes of 'non-NHS' dental care compared with dental care provided within the National Health Service.

Dr Nigel Nuttall, Reader, Dental Health Services Research Unit, Dundee Dental Hospital and School, University of Dundee 